Article

\title{
Electronic Structure and Maximum Energy Product of MnBi
}

\author{
Jihoon Park ${ }^{1}$, Yang-Ki Hong ${ }^{1, *}$, Jaejin Lee ${ }^{1}$, Woncheol Lee ${ }^{1}$, Seong-Gon Kim ${ }^{2}$ and \\ Chul-Jin Choi ${ }^{3}$
}

1 Department of Electrical and Computer Engineering and MINT Center, the University of Alabama, Tuscaloosa, AL 35487, USA; E-Mails: jpark61@crimson.ua.edu (J.P.); jjlee@crimson.ua.edu (J.L.); wlee43@crimson.ua.edu (W.L.)

2 Department of Physics \& Astronomy and Center for Computational Sciences, Mississippi State University, Starkville, MS 39792, USA;

E-Mail: seong-gon.kim@msstate.edu

3 Korea Institute of Materials Science, Changwon, Kyungsangnam-do 642-831, Korea; E-Mail: cjchoi@kims.re.kr

* Author to whom correspondence should be addressed; E-Mail: ykhong@eng.ua.edu; Tel.: +1-205-348-7268; Fax: +1-205-348-6959.

Received: 30 June 2014; in revised form: 20 August 2014 / Accepted: 21 August 2014 / Published: 29 August 2014

\begin{abstract}
We have performed first-principles calculations to obtain magnetic moment, magnetocrystalline anisotropy energy (MAE), i.e., the magnetic crystalline anisotropy constant $(K)$, and the Curie temperature $\left(T_{\mathrm{c}}\right)$ of low temperature phase (LTP) MnBi and also estimated the maximum energy product $(B H)_{\max }$ at elevated temperatures. The full-potential linearized augmented plane wave (FPLAPW) method, based on density functional theory (DFT) within the local spin density approximation (LSDA), was used to calculate the electronic structure of LPM MnBi. The $T_{\mathrm{c}}$ was calculated by the mean field theory. The calculated magnetic moment, MAE, and $T_{\mathrm{c}}$ are $3.63 \mu_{\mathrm{B}} /$ f.u. (formula unit) (79 emu/g or $714 \mathrm{emu} / \mathrm{cm}^{3}$ ), $-0.163 \mathrm{meV} /$ u.c. (or $K=-0.275 \times 10^{6} \mathrm{~J} / \mathrm{m}^{3}$ ) and $711 \mathrm{~K}$, respectively. The $(B H)_{\max }$ at the elevated temperatures was estimated by combining experimental coercivity $\left(H_{\mathrm{ci}}\right)$ and the temperature dependence of magnetization $\left(M_{S}(T)\right)$. The $(B H)_{\max }$ is 17.7 MGOe at $300 \mathrm{~K}$, which is in good agreement with the experimental result for directionally-solidified LTP MnBi (17 MGOe). In addition, a study of electron density maps and the lattice constant $c / a$ ratio dependence of the magnetic moment suggested that doping of a third element into interstitial sites of LTP MnBi can increase the $M_{s}$.
\end{abstract}


Keywords: MnBi; permanent magnet; first-principles calculation; magnetization; anisotropy constant; maximum energy product

\section{Introduction}

The maximum energy product $(\mathrm{BH})_{\max }$ of a permanent magnet at elevated temperatures has become increasingly important because a motor for electric vehicles (EV) should be operational at $473 \mathrm{~K}$. Rare-earth Nd-Fe-B magnets show the highest $(B H)_{\max }$ of $59 \mathrm{MGOe}$ at room temperature [1], which is much larger than 5.4 MGOe for the most widely used low energy ferrite magnets [2]. Although the Nd-Fe-B magnets exhibit desirable magnetic flux density $(B)$, intrinsic coercivity $\left(H_{c i}\right.$ and $\left.(B H)_{\max }\right)$, a large negative temperature coefficient of $H_{c i}$, a low Curie temperature $\left(T_{\mathrm{c}}\right)$ of $523 \mathrm{~K}$ [3] and limited supply are the critical drawbacks that need to be addressed. Motors for electric vehicles (EV) must reliably operate at temperatures up to $473 \mathrm{~K}$. Therefore, the usage of Nd-Fe-B magnets is limited to a lower temperature than $473 \mathrm{~K}$, due to their negative temperature coefficient of $H_{\mathrm{ci}}$ and low Curie temperature. On the other hand, low temperature phase (LTP) MnBi shows a positive magnetic anisotropy coefficient [4-6] resulting in $H_{c i}$ of about $1.5 \mathrm{~T}$ at $300 \mathrm{~K}$ and $2 \mathrm{~T}$ at $400 \mathrm{~K}$ [5]. The high $H_{c i}$ of LTP MnBi helps to make it usable at the operating temperature of the motor. The directionally-solidified LTP MnBi shows a remanent magnetic flux density $\left(B_{r}\right)$ of $0.8 \mathrm{~T}$ and $(B H)_{\max }$ of $17 \mathrm{MGOe}$ at $290 \mathrm{~K}$ [7]. However, arc-melted and mechanically-milled LTP MnBi powder shows a low $B_{r}$ of $0.7 \mathrm{~T}$ and $(B H)_{\max }$ of 11.00 MGOe [8] and $B_{r}$ of $0.7 \mathrm{~T}$ and $(B H)_{\max }$ of 11.95 MGOe [9] at $300 \mathrm{~K}$. Therefore, it is imperative to predict the theoretical limit of $(B H)_{\max }$ for the LTP MnBi magnet.

Coehoorn et al. performed first self-consistent spin-polarized band-structure calculations and obtained a magnetic moment of $3.6 \mu_{\mathrm{B}}$, the density of states (DOS) and the band structures at $0 \mathrm{~K}$ [10]. Köhler et al. used first-principles calculation based on local density functional approximation (LDA) and the augmented spherical wave (ASW) band structure method to calculate the magneto-optical properties, a magnetic moment of $3.61 \mu_{\mathrm{B}}$, a magnetocrystalline anisotropy constant $(K)$ of $1.5 \times 10^{6} \mathrm{~J} / \mathrm{m}^{3}$, DOS and the band structures at $0 \mathrm{~K}$ [11]. Furthermore, Huberman et al. have predicted the normalized temperature dependence of magnetic moment within the mean-field theory [12]. However, the theoretical limit of saturation magnetization $\left(M_{\mathrm{s}}\right)$, and thereby $(B H)_{\max }$, at elevated temperatures and the possibility of an increase in the $M_{\mathrm{s}}$ has not been reported.

In this paper, we calculated DOS, and, thereby, the magnetic moment, and $K$ using first-principles calculations and $T_{c}$ by the mean field theory. Then, we have used these parameters to determine the temperature dependence of saturation magnetization $M_{s}(T)$ and the maximum energy product $(B H)_{\max }(T)$. In addition, an electron density map was obtained to find if any interstitial site is available for alloying elements. The lattice constant $c / a$ ratio dependence of magnetic moment was investigated to explore a possible increase in $M_{s}$.

\section{Method of Calculations}

The WIEN2k package [13] was used to perform first-principles calculations. The package is based on density functional theory (DFT) and uses the full-potential linearized augmented plane wave 
(FPLAPW) method with the dual basis set. For the LTP MnBi, the $3 s, 3 p, 3 d$ and $4 s$ states of Mn and the $5 p, 5 d, 6 s$ and $6 p$ states of $\mathrm{Bi}$ were taken as valence states. All calculations used a $25 \times 25 \times 15$ k-point mesh generating 1,352 k-points in the irreducible part of the Brillouin zone. The muffin tin radii (RMT) of 2.50 a.u. for $\mathrm{Mn}$ and 2.23 a.u. for $\mathrm{Bi}$ and the experimental lattice constants $a=4.256 \AA$ and $c=6.042 \AA$ at $4.2 \mathrm{~K}[6]$ were used.

The density functional theory within the local-spin-density approximation (LSDA) was used for all spin-polarized and spin-orbit coupling calculations. Spin and orbital magnetic moments were calculated for the magnetization directions of $<100>$ and $<001>$. The total energies calculated for these two different magnetization directions were used to obtain $K$.

\section{Results and Discussion}

\subsection{Density of States and Magnetic Moments}

Figure 1a,b shows the density of states (DOS) in $<100>$ and $<001>$ magnetization directions of the LTP MnBi, which were calculated based on spin-polarized and spin-orbit coupling. The DOS is divided into lower and higher energy regions. The lower energy region near -0.4 Ry is mostly contributed by the $s$ band of $\mathrm{Bi}$, and the higher energy region near the Fermi energy $\left(E_{\mathrm{F}}\right)$ is mostly contributed by the $d$ band of Mn, as seen in Figure 1a,b. The DOS near the $E_{\mathrm{F}}$ is a highly-degenerated energy state; slightly below the $E_{\mathrm{F}}$ in the majority spin state and slightly above the $E_{\mathrm{F}}$ in the minority spin state. These highly-degenerate energy states near $E_{\mathrm{F}}$ are the origin of the magnetic moment of LTP MnBi by contributing to the difference between the number of electrons in the majority and minority spin states below $E_{\mathrm{F}}$. The spin and orbital magnetic moments, which were calculated from the spin polarized and spin-orbit couplings, are given in Table 1. It is noted that the orbital magnetic moment from spin-orbit coupling calculations in the $<100>$ magnetization direction is higher than that of the $<001>$ magnetization direction. This difference results in the highest total magnetic moment of $3.63 \mu_{\mathrm{B}} /$ f.u. (formula unit) (79 emu/g or $714 \mathrm{emu} / \mathrm{cm}^{3}$ ) for LTP MnBi. This magnetic moment value is in good agreement with the results of first-principles calculations based on local density functional approximation (LDA) and the augmented spherical wave (ASW) band structure method (3.61 $\left.\mu_{\mathrm{B}}\right)$ [11] and first self-consistent spin-polarized band-structure calculations (3.6 $\left.\mu_{\mathrm{B}}\right)$ [10]. However, this calculated value is smaller than the experimental neutron diffraction measurement value of $3.8 \mu_{\mathrm{B}}$ at $300 \mathrm{~K}[6]$ and the extrapolated value of $3.9 \mu_{\mathrm{B}}$ at $0 \mathrm{~K}$ [14].

Table 1. The calculated spin and orbital magnetic moments per formula unit (f.u.) and Mn and $\mathrm{Bi}$ atoms for low temperature phase (LTP) $\mathrm{MnBi}$ in units of $\mu_{\mathrm{B}}$. Magnetic moments from both spin-polarized and spin-orbit coupling calculations are listed.

\begin{tabular}{ccccccccc}
\hline \multirow{2}{*}{$\begin{array}{c}\text { Direction of } \\
\text { magnetization }\end{array}$} & \multicolumn{2}{c}{ Spin moment } & & \multicolumn{2}{c}{ Orbital moment } & & \multicolumn{2}{c}{ Total moment } \\
\cline { 2 - 3 } & f.u. & $\mathbf{M n} / \mathbf{B i}$ & & f.u. & $\mathbf{M n} / \mathbf{B i}$ & & f.u. & $\mathbf{M n} / \mathbf{B i}$ \\
\hline Spin-polarized & 3.50 & $3.57 /-0.07$ & & & & & 3.50 & $3.57 /-0.07$ \\
$<100>$ & 3.52 & $3.5 /-0.06$ & & 0.105 & $0.10595 /-0.00009$ & & 3.63 & $3.68 /-0.06$ \\
$<001>$ & 3.52 & $3.58 /-0.06$ & & 0.084 & $0.08415 /-0.00012$ & & 3.60 & $3.66 /-0.06$ \\
\hline
\end{tabular}


Figure 1. Density of states (DOS) for LTP MnBi in the (a) $<100>$ and (b) $<001>$ magnetization directions. The black line represents the total DOS, and blue and pink lines represent the partial DOS of $\mathrm{Mn}$ and Bi, respectively. The red vertical line corresponds to the Fermi energy $\left(E_{\mathrm{F}}\right)$.

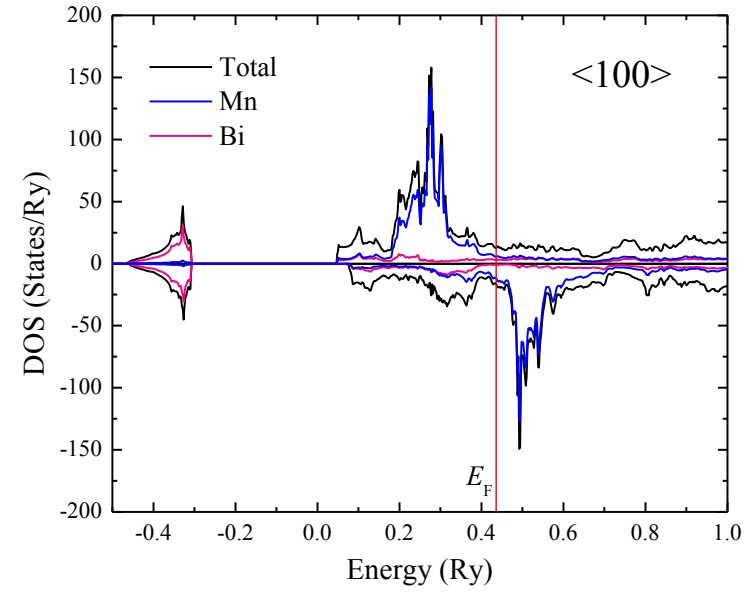

(a)

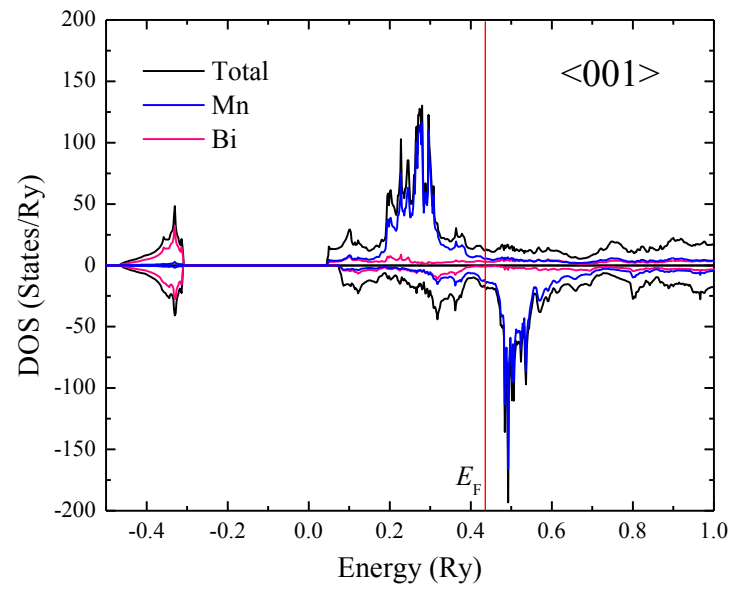

(b)

\subsection{Magnetocrystalline Anisotropy Constant}

Magnetocrystalline anisotropy energy (MAE) is the total energy difference between $<100>$ and $<001>$ magnetization directions, i.e., $\Delta E=E_{<100>}-E_{<001>}$. The calculated total energy difference is $-0.163 \mathrm{meV} /$ u.c., which corresponds to an in-plane $K$ of $-0.275 \times 10^{6} \mathrm{~J} / \mathrm{m}^{3}$ at $0 \mathrm{~K}$. The DOS in Figure 1a,b, and the calculated $K$ imply that forcing the magnetization direction from the easy plane to the $c$-axis results in destruction of the degeneracy near the $E_{\mathrm{F}}$, thereby increasing the total energy. The destruction of degeneracy (lower and broader DOS peaks) can be observed by comparing the DOS of $<100>$ and $<001>$ magnetization directions below the $E_{\mathrm{F}}$. Our calculated $K$ is close to the experimental $K$ of $-0.155 \times 10^{6} \mathrm{~J} / \mathrm{m}^{3}$ at $4.2 \mathrm{~K}$ [6], but smaller than the reported $K$ value of $1.5 \times 10^{6} \mathrm{~J} / \mathrm{m}^{3}$ [11], indicating a strong anisotropy along with $c$-axis. It was experimentally observed that the spin rotated from the basal plane to the $c$-axis at about $90 \mathrm{~K}$ [15]. The $K$ was measured in the range of 4.2 to $628 \mathrm{~K}$ [4] using a vibrating sample magnetometer (VSM) and by singular point detection (SPD) from 147 to $586 \mathrm{~K}$ [5]. The measured $K$ is $1.3 \times 10^{6} \mathrm{~J} / \mathrm{m}^{3}$ [4] and $1.8 \times 10^{6} \mathrm{~J} / \mathrm{m}^{3}$ [5] at $300 \mathrm{~K}$ and $2.3 \times 10^{6} \mathrm{~J} / \mathrm{m}^{3}$ at $430 \mathrm{~K}$ [5], suggesting out-of-plane magnetization at the elevated temperatures.

\subsection{Curie Temperature}

In this section, the $T_{\mathrm{c}}$ for LTP MnBi was calculated using the following mean field approximation (MFA) expression [16]:

$$
T_{c}=\frac{2}{3 k_{B}} J_{0} \gamma, J_{0}=\sum_{j} J_{0 j}
$$

where $J_{0}$ is the molecular field parameter calculated by summing the exchange integrals $\left(J_{0 j}\right)$ and $k_{\mathrm{B}}$ is the Boltzmann constant. The factor $\gamma$ is given by $S(S+1) / S^{2}$ for quantum spins and one for classical 
spins. In this work, we choose $\gamma=1$, which gives a $T_{\mathrm{c}}$ close to the experimental one. This is because the temperature dependences of $J_{0 j}$ and $S$ are mutually cancelled [17,18]. The $J_{0 j}$ are obtained by the exchange energy difference between the ground and excited states of sublattices. The exchange integral [15] is written as:

$$
J_{i j}=\left(\Delta_{i j}-\Delta_{i}-\Delta_{j}\right) /\left(4 S_{i} S_{j} n_{i} z_{i j}\right)
$$

where $\Delta_{i j}$ is the difference of exchange energies between the excited state at both the $i$ and $j$ sublattices and the ground state, $\Delta_{i}$ is the difference between the excited state at the $i$ sublattice and the ground state, $\Delta_{j}$ is the difference between the excited state at the $j$ sublattice and the ground state, $S_{i}$ is the spin of the $i$-th $\mathrm{Mn}$ atom, $n_{i}$ is the number of $i$-th $\mathrm{Mn}$ atoms and $z_{i j}$ is the number of neighboring $j$-th $\mathrm{Mn}$ atoms to $i$-th $\mathrm{Mn}$ atom. The exchange interaction between spins is inversely and exponentially proportional to the corresponding distance. The distance and number of corresponding neighbors for LTP MnBi are given in Table 2. The distance of the third and fourth nearest neighbors is greater than $0.5 \mathrm{~nm}$. Therefore, the exchange interactions between these spins are negligible. Accordingly, the energy difference $\Delta$ between the ground and exited states was calculated only for the first and second nearest neighbors. To include the significant exchange interaction with the nearest neighbors, the supercell structures (two LTP MnBi unit cells) were built. The input parameter $S$ and $n_{i}$ for LTP MnBi are two and one, respectively. The $z_{i j}$ values for the first and second nearest neighbors were taken from Table 2.

Table 2. The number of nearest neighbors $\left(z_{0 \mathrm{i}}\right)$ and corresponding distances $\left(r_{0 \mathrm{i}}\right)$ in the unit of $\AA$ in LTP MnBi.

\begin{tabular}{ccccccccccc}
\hline \multirow{2}{*}{ Material } & \multicolumn{3}{c}{ \# of nearest neighbors } & & \multicolumn{4}{c}{ Distance } \\
\cline { 2 - 5 } \cline { 7 - 10 } & $\boldsymbol{z}_{\mathbf{0 1}}$ & $\boldsymbol{z}_{\mathbf{0 2}}$ & $\boldsymbol{z}_{\mathbf{0 3}}$ & $\boldsymbol{z}_{\mathbf{0 4}}$ & & $\boldsymbol{r}_{\mathbf{0 1}}$ & $\boldsymbol{r}_{\mathbf{0 2}}$ & $\boldsymbol{r}_{\mathbf{0 3}}$ & $\boldsymbol{r}_{\mathbf{0 4}}$ \\
\hline LTP MnBi & 2 & 6 & 12 & 2 & & 3.02 & 4.26 & 5.22 & 6.04 \\
\hline
\end{tabular}

As a result, the calculated first and second exchange integrals $\left(J_{01}\right.$ and $\left.J_{02}\right)$ are 32.31 and $4.59 \mathrm{meV}$, respectively, which corresponds to the mean-field estimated $T_{\mathrm{c}}=711 \mathrm{~K}$. Experimentally, the LTP $\mathrm{MnBi}$ structure transforms to the high temperature phase (HTP) $\mathrm{MnBi}$ at $628 \mathrm{~K}$ [4]; thereby, a dramatic drop of magnetization occurs at $628 \mathrm{~K}$.

\subsection{Temperature Dependence of Saturation Magnetization and Maximum Energy Product}

We use the mean field theory to calculate $M_{\mathrm{s}}(T)$. The $M_{\mathrm{s}}(T)$ within the MFA [19] is given by:

$$
M_{s}(T)=M_{s}(0) B_{J}(\tau)
$$

where $M_{s}(0)$ is the saturation magnetization at $0 \mathrm{~K}, B_{J}$ is the Brillouin function with angular quantum number $(J)$ and normalized temperature $\left(\tau=T / T_{c}\right)$. $J$ is the summation of spin moment $(S)$ and orbital contribution $(L)$. The calculated magnetic moment of $3.63 \mu_{\mathrm{B}} /$ f.u., $T_{\mathrm{c}}$ of $711 \mathrm{~K}$ and $J$ of two were used to calculate the $M_{s}(T)$. Figure 2 shows the calculated and experimental [5] $M_{s}(T)$. The calculated values are in good agreement with the experimental data. 
Figure 2. The calculated and experimental temperature dependence of saturation magnetization $M_{s}(T)$ for LTP MnBi.

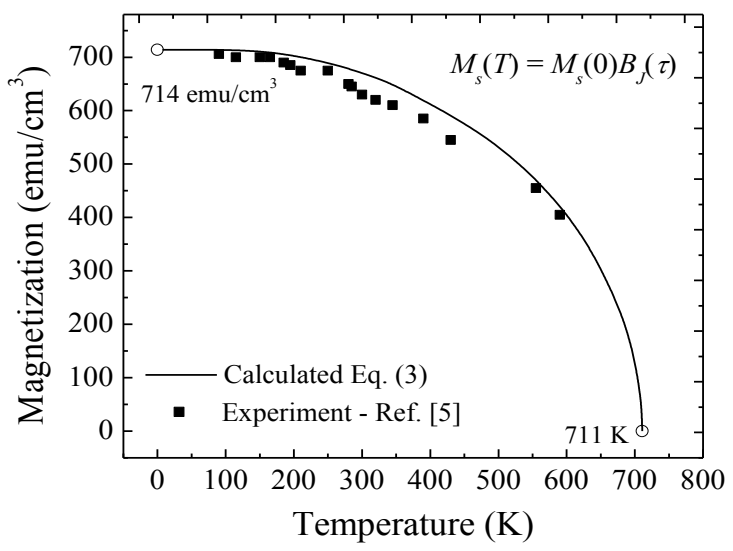

Therefore, we used the calculated $M_{s}(T)$ to estimate the temperature dependence of the maximum energy product $(B H)_{\max }(T)$ for the LTP MnBi. In order to calculate the $(B H)_{\max }(T)$, the temperature dependence of intrinsic coercivity $H_{c i}(T)$ is also needed. We used the experimental $H_{c i}(T)$ [15] in Figure 3 and assume coherent magnetization reversal and a rectangular hysteresis loop of LTP MnBi to estimate $(B H)_{\max }(T)$. The $(B H)_{\max }$ is the maximum amount of magnetic energy stored in a magnet, which is the maximum rectangular area in the $B-H$ loop. Therefore, the $(B H)_{\max }(T)$ can be obtained by either Equation (4) or (5).

$$
\begin{gathered}
(B H)_{\max }(T)=\left[B_{r}(T)-H_{c i}(T)\right] H_{c i}(T) \\
(B H)_{\max }(T)=\frac{B_{r}^{2}(T)}{4}
\end{gathered}
$$

where $B_{r}$ is the remnant magnetic flux density. Equation (4) is used for the case of $H_{c i}<B_{r} / 2$, and Equation (5) is for $H_{c i}>B_{r} / 2$. From a comparison between Figures 2 and 3, the $H_{c i}$ is smaller than $B_{r} / 2$ below $250 \mathrm{~K}$, while it is greater than $B_{r} / 2$ above $250 \mathrm{~K}$ for LTP MnBi.

Figure 3. Experimental intrinsic coercivity $\left(H_{\mathrm{ci}}\right)$ as a function of temperature for LTP MnBi.

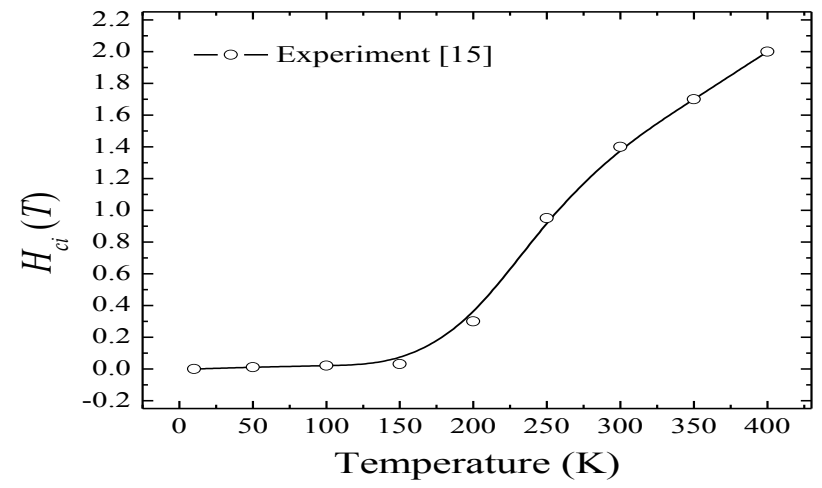

Therefore, Equation (4) was used below $250 \mathrm{~K}$, and Equation (5) was used above $250 \mathrm{~K}$ to estimate $(B H)_{\max }(T)$. Figure 4 shows the semi-theoretical $(B H)_{\max }(T)$ and experimental $(B H)_{\max }[8,9,13,20]$ for LTP MnBi, including experimental $(B H)_{\max }(T)$ for Nd-Fe-B [3]. It is noted that the $(B H)_{\max }$ of LTP 
MnBi dramatically increased from 1.7 MGOe at $100 \mathrm{~K}$ to $18.7 \mathrm{MGOe}$ at $250 \mathrm{~K}$ and then gradually decreased to $6.2 \mathrm{MGOe}$ at $600 \mathrm{~K}$. The calculated $(B H)_{\max }$ at $300 \mathrm{~K}$ is $17.7 \mathrm{MGOe}$, and it is in good agreement with the $(B H)_{\max }$ of directionally-solidified LTP MnBi (17 MGOe at $290 \mathrm{~K}$ [7]). However, it is larger than those of arc-melted and mechanically-milled $\mathrm{MnBi}$, which are $11 \mathrm{MGOe}$ [8] for powder and 11.9 MGOe for a powder-wax mixture [9] at $300 \mathrm{~K}$. Resin-bonded MnBi magnets show $(B H)_{\max }$ values of 7.7 and 4.6 MGOe at 300 and $400 \mathrm{~K}$, respectively [15], and a sintered MnBi magnet shows $(B H)_{\max }$ values of $7.8 \mathrm{MGOe}$ [9] and 5.8 MGOe [20] at $300 \mathrm{~K}$. All of these $(B H)_{\max }$ are smaller than the calculated values due to the imperfect rectangular demagnetization curve. As shown in Figure 4, the semi-theoretical $(B H)_{\max }$ of LTP MnBi (10.9 MGOe) was found to be higher than that of $\mathrm{Nd}-\mathrm{Fe}-\mathrm{B}(2 \mathrm{MGOe})$ at $500 \mathrm{~K}$ [3]. This result suggests that LTP MnBi is a good permanent magnet for high temperature applications.

Figure 4. The calculated temperature dependence of the maximum energy product $(B H)_{\max }$ $(T)$ and experimental $(B H)_{\max }$ for LTP MnBi and experimental $(B H)_{\max }(T)$ for Nd-Fe-B.

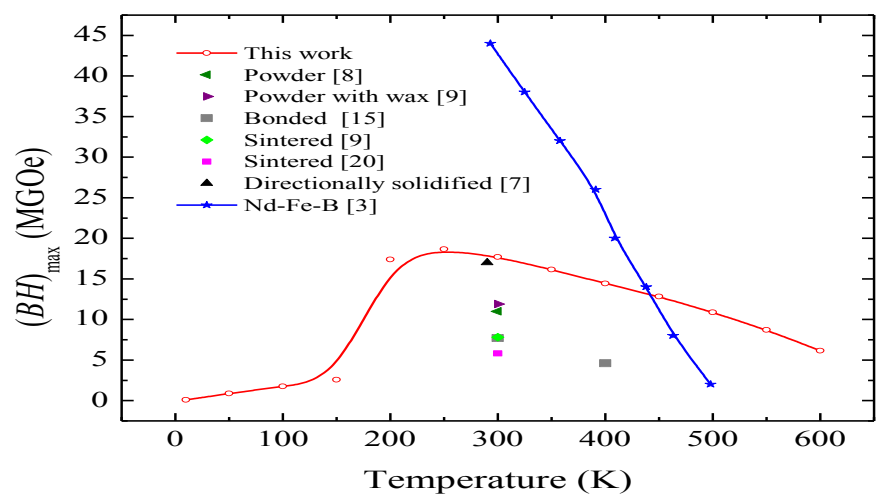

\subsection{Electron Density Maps and c/a Ratio Dependence of Magnetic Moment}

In this section, we discuss the effect of the doping element on the possibility of an increase in the $M_{\mathrm{s}}$ of LTP MnBi, and thereby $(B H)_{\max }$. Figure 5 shows the $c / a$ ratio dependence of the total magnetic moment and relative total energy with a constant volume. As the distance between $\mathrm{Mn}$ atoms in the $c$-axis increases, the magnetic moment increases, and the total energy decreases up to a $c / a$ ratio of about 1.37 and then increases. The magnetic moment at this ratio is $3.63 \mu_{\mathrm{B}} /$ f.u., which is in good agreement with the above calculated moment.

Figure 5. The $c / a$ dependence of magnetic moment and relative total energy for $\mathrm{MnBi}$.

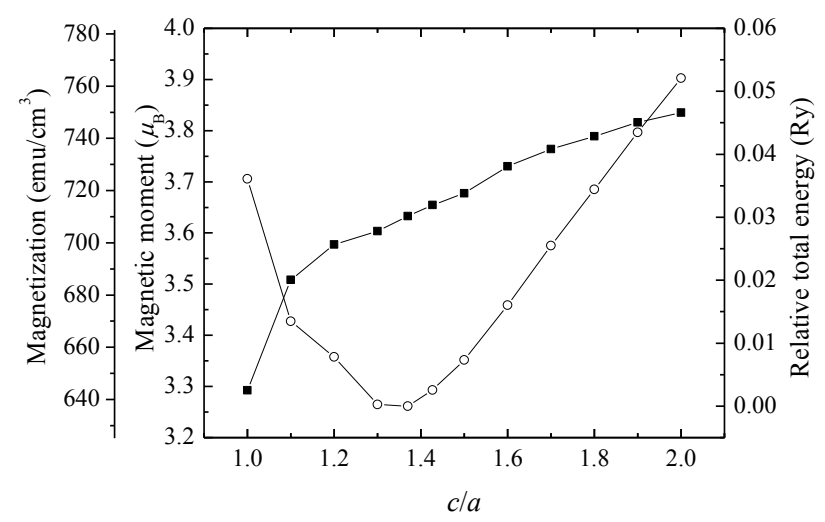


In order to explore any possibility of increasing the magnetic moment, we calculated the electron density and, thereby, find interstitial sites available for the doping element. Figure 6 shows the three- (3D) and two-dimensional (2D) electron density maps for (110), (100) and (001) planes. The 2 -D electron density maps show that the positions of $(0.667,0.333,0.250)$ and $(0.333,0.667,0.750)$ have low electron density areas. Therefore, these sites can be interstitial sites for alloying elements.

Figure 6. The 3D and 2D electron density maps for LTP MnBi in (a) (110), (b) (100) and (c) (001) planes. The peaks colors, and contour lines indicate the electron density.

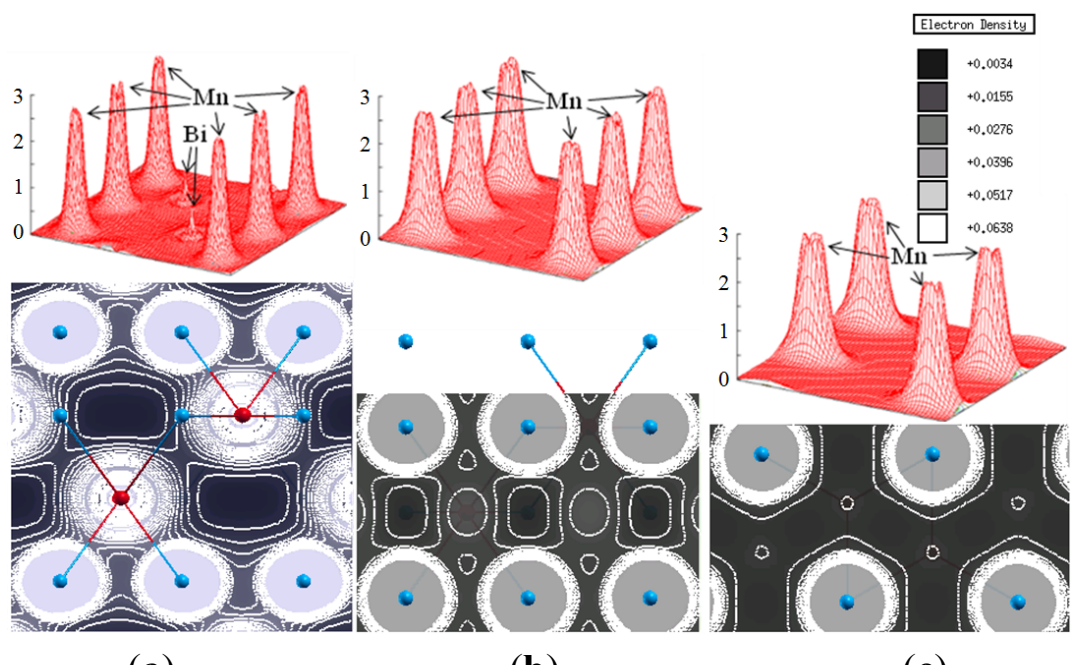

(a)

(b)

(c)

The identified interstitial sites in the LTP MnBi structure are shown in Figure 7. From these results, we can conclude that doping with a third element into the interstitial sites of LTP MnBi is feasible. In this way, it could be possible to increase the $c / a$ ratio, thereby increasing $M_{\mathrm{s}}$. We previously reported that the ternary and quaternary systems of MnBi-Co and MnBi-Co-Fe show increased $M_{s}$ values of $730 \mathrm{emu} / \mathrm{cm}^{3}$ and $810 \mathrm{emu} / \mathrm{cm}^{3}$, respectively, as compared to $714 \mathrm{emu} / \mathrm{cm}^{3}$ for LTP MnBi [21]. Moreover, it was found that the $K$ increased from $-0.275 \times 10^{6} \mathrm{~J} / \mathrm{m}^{3}$ for LTP MnBi to $1.9 \times 10^{6} \mathrm{~J} / \mathrm{m}^{3}$ for MnBi-Co and $1.62 \times 10^{6} \mathrm{~J} / \mathrm{m}^{3}$ for MnBi-Co-Fe. These results imply that doping of the elements into the interstitial sites changes the weak easy plane anisotropy of LTP MnBi to the strong easy axis anisotropy at $0 \mathrm{~K}[21]$.

Figure 7. Yellow boxes indicate the low electron density areas of the hexagonal LTP MnBi structure.

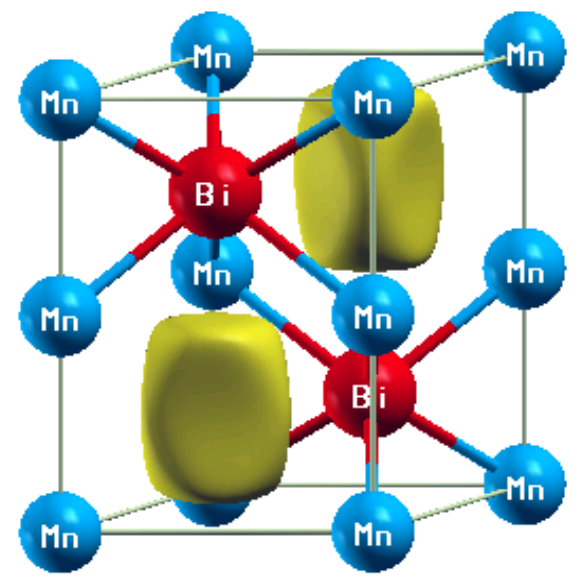


Although the $M_{s}$ and $K$ are enhanced by the doping, the $T_{\mathrm{c}}$ decreases to $325 \mathrm{~K}$ for MnBi-Co and $275 \mathrm{~K}$ for MnBi-Co-Fe [21] from $711 \mathrm{~K}$ for binary $\mathrm{MnBi}$. These low $T_{\mathrm{c}}$ are the drawbacks of the Co and Co-Fe-doped MnBi-based permanent magnets, which precludes high temperature applications. It is noted that a structural transition by migration of $\mathrm{Mn}$ atoms to the interstitial sites occurs at a high temperature, which causes anti-ferromagnetic coupling between Mn atoms [22]. The doping of a third element into the interstitial sites possibly prevents LTP MnBi from the structural transition, thereby maintaining the high $T_{\mathrm{c}}$.

\section{Conclusions}

The calculated magnetic moment, MAE, and $T_{\mathrm{c}}$ are $3.63 \mu_{\mathrm{B}} / \mathrm{f} . \mathrm{u} .\left(79 \mathrm{emu} / \mathrm{g}\right.$ or $\left.714 \mathrm{emu} / \mathrm{cm}^{3}\right)$, $-0.163 \mathrm{meV} /$ u.c. (or $K=-0.275 \times 10^{6} \mathrm{~J} / \mathrm{m}^{3}$ ) and $711 \mathrm{~K}$, respectively. It was found that the $(B H)_{\max }$ at $300 \mathrm{~K}$ is $17.7 \mathrm{MGOe}$, which is in good agreement with the experimental result reported for directionally-solidified LTP MnBi (17 MGOe). The study of the electron density maps and $c / a$ ratio dependence of the magnetic moment suggested that doping of a third element into interstitial sites of LTP MnBi can increase the $M_{s}$. The LTP MnBi outperforms Nd-Fe-B above $450 \mathrm{~K}$.

\section{Acknowledgments}

This work was supported in part by the U.S. Department of Energy Advanced Research Projects Agency-Energy Rare Earth Alternatives in Critical Technologies (ARPA-E REACT) Program under Award Number DE-AR0000189, National Science Foundation-The Civil, Mechanical and Manufacturing Innovation (NSF-CMMI) under Award Number 1229049 and the E. A. "Larry" Drummond Endowment at the University of Alabama.

\section{Conflicts of Interests}

The authors declare no conflicts of interests.

\section{References}

1. Sugimoto, S. Current status and recent topics of rare-earth permanent magnets. J. Phys. D: Appl. Phys. 2011, 44, 064001-064011.

2. Hitachi Ferrite Catalog 2007. Available online: http://www.hitachimetals.com/product/ permanentmagnets/ferrite/ (accessed on 27 August 2014).

3. Akiya, T.; Kato, H.; Sagawa, M.; Koyama, K. Enhancement of coercivity in Al and Cu added Nd-Fe-B sintered magnets by high field annealing. Mater. Sci. Eng. 2009, 1, 012034-012039.

4. Chen, T.; Stutius, W.E. The phase transformation and physical properties of the MnBi and $\mathrm{Mn}_{1.08} \mathrm{Bi}$ compounds. IEEE Trans. Magn. 1974, 10, 581-586.

5. Guo, X.; Chen, X.; Altounian, Z.; Ström-Olsen, J.O. Magnetic properties of MnBi prepared by rapid solidification. Phys. Rev. B 1992, 46, 14578-14582.

6. Roberts, B.W. Neutron diffraction study of the structures and magnetic properties of manganese bismuthide. Phys. Rev. 1956, 104, 607-616. 
7. Pirich, R.G.; Larson, D.J. Directional solidification and densification of permanent magnets having single domain size MnBi particles. U.S. Patent US4,784,703, 15 November 1988.

8. Chinnasamy, C.; Jasinski, M.M.; Ulmer, A.; Li, W.; Hadjipanayis, G.; Liu, J. Mn-Bi magnetic powders with high coercivity and magnetization at room temperature. IEEE Trans. Magn. 2012, 48, 3641-3643.

9. Cui, J.; Choi, J.P.; Li, G.; Polikarpov, E.; Darsell, J.; Overman, N.; Olszta, M.; Schreiber, D.; Bowden, M.; Droubay, T.; et al. Thermal stability of MnBi magnetic materials. J. Phys.: Condens. Matter 2014, 26, 064212-064221.

10. Coehoorn, R.; de Groot, R.A. The electronic structure of MnBi. J. Phys. F: Met. Phys. 1985, 15, 2135-2144.

11. Köhler, J.; Kübler, J. Calculated magneto-optical properties of pure and doped MnBi. J. Phys.: Condens. Matter 1996, 8, 8681-8694.

12. Huberman, B.A.; Streifer, W. Coupled order parameters, lattice disorder, and magnetic phase transition. Phys. Rev. B 1975, 12, 2741-2746.

13. Blaha, P.; Schwarz, K.; Madsen, G.; Kvasnicka, D.; Luitz, J. WIEN2k: An Augmented Plane Wave + Local Orbitals Program for Calculating Crystal Properties; Karlheinz Schwarz Techn. Universität Wien: Vienna, Austria, 2001.

14. Heikes, R.R. Magnetic Transformation in MnBi. Phys. Rev. 1955, 99, 446-447.

15. Yang, J.B.; Yelon, W.B.; James, W.J.; Cai, Q.; Kornecki, M.; Roy, S.; Ali, N.; 1'Heritier, Ph. Crystal structure, magnetic properties and electronic structure of the MnBi intermetallic compound. J. Phys.: Condens. Matter 2002, 14, 6509-6519.

16. MacLaren, J.M.; Schulthess, T.C.; Butler, W.H.; Sutton, R.; McHenry, M. Electronic structure, exchange interactions, and Curie temperature of FeCo. J. Appl. Phys. 1999, 85, 4833-4835.

17. Gyorffy, B.L.; Pindor, A.J.; Staunton, J.; Stocks, G.M.; Winter, H. A first-principles theory of ferromagnetic phase transitions in metals. J. Phys. F 1985, 15, 1337-1386.

18. Liechtenstein, A.I.; Katsnelson, M.I.; Antropov, V.P.; Gubanov, V.A. Local spin excitations and curie temperature of iron. J. Magn. Magn. Mater. 1987, 67, 65-74.

19. Cullity, B.D.; Graham, C.D. Introduction to Magnetic Materials, 2nd ed.; Wiley: Hoboken, NJ, USA, 2009; p. 121.

20. Rao, N.V.R.; Gabay, A.M.; Hadjipanayis, G.C. Anisotropic fully dense MnBi permanent magnet with high energy product and high coercivity at elevated temperatures. J. Phys. D: Appl. Phys. 2013, 46, 062001-062004.

21. Hong, Y.K.; Park, J.H.; Mryasov, O.N.; Kim, S.G.; Kim, S.H.; Lee, J.J.; Abo, G.S.; Choi, C.J.; Lee, J.G. Magnetic properties of MnBi based alloys: First-principles calculations for MnBi-Co and MnBi-Co-Fe cases. AIP Advances 2013, 3, 052137-052141.

22. Bandaru, P.R.; Sands, T.D.; Kubota, Y.; Marinero, E. Decoupling the structural and magnetic phase transformations in magneto-optic MnBi thin films by the partial substitution of $\mathrm{Cr}$ for $\mathrm{Mn}$. Appl. Phys. Lett. 1998, 72, 2337-2339.

(C) 2014 by the authors; licensee MDPI, Basel, Switzerland. This article is an open access article distributed under the terms and conditions of the Creative Commons Attribution license (http://creativecommons.org/licenses/by/3.0/). 